\title{
On joint distributions of order statistics for a discrete case
}

\author{
M Güngör ${ }^{1 *}$, Y Bulut ${ }^{2}$ and B Yüzbaşı ${ }^{1}$
}

"Correspondence: mgungor44@gmail.com

${ }^{1}$ Department of Econometrics, Inonu University, Malatya, 44280, Turkey

Full list of author information is available at the end of the article

\begin{abstract}
In this study, the joint distributions of order statistics of innid discrete random variables are expressed. Also, the joint distributions are obtained in the form of an integral. Then, the results related to $p f$ and $d f$ are given.
\end{abstract}

MSC: $62 \mathrm{G} 30 ; 62 \mathrm{E} 15$

Keywords: order statistics; discrete random variable; probability function; distribution function

\section{Introduction}

The joint probability density function $(p d f)$ and marginal $p d f$ of order statistics of independent but not necessarily identically distributed (innid) random variables was derived by Vaughan and Venables [1] by means of permanents. In addition, Balakrishnan [2] and Bapat and Beg [3] obtained the joint $p d f$ and distribution function $(d f)$ of order statistics of innid random variables by means of permanents. Balasubramanian et al. [4] obtained the distribution of single order statistics in terms of distribution functions of the minimum and maximum order statistics of some subsets of $\left\{X_{1}, X_{2}, \ldots, X_{n}\right\}$ where $X_{i}$ 's are innid random variables. Later, Balasubramanian et al. [5] generalized their previous results [4] to the case of the joint distribution function of several order statistics. Recurrence relationships among the distribution functions of order statistics arising from innid random variables were obtained by Cao and West [6]. Using multinomial arguments, the $p d f$ of $X_{r: n+1}(1 \leq r \leq n+1)$ was obtained by Childs and Balakrishnan [7] by adding another independent random variable to the original $n$ variables $X_{1}, X_{2}, \ldots, X_{n}$. Also, Balasubramanian et al. [8] established the identities satisfied by the distributions of order statistics from non-independent non-identical variables through operator methods based on the difference and differential operators. In 1991, Beg [9] obtained several recurrence relations and identities for product moments of order statistics of innid random variables using permanents. Recently, Cramer et al. [10] derived the expressions for the distribution and density functions by Ryser's method and the distributions of maxima and minima based on permanents.

The notion of distribution theory has been applied in various branches of science for investigations. Recently, the notion of a uniform distribution has been applied in sequence spaces and the notion of statistical convergent sequences has been studied and investigated from different aspects by Rath and Tripathy [11], Tripathy [12, 13], Tripathy and Baruah [14], Tripathy and Dutta [15], Tripathy and Sarma [16], Tripathy and Sen [17], and others.

() 2012 Güngör et al.: licensee Springer. This is an Open Access article distributed under the terms of the Creative Commons Attribution License (http://creativecommons.org/licenses/by/2.0), which permits unrestricted use, distribution, and reproduction in any medium, provided the original work is properly cited. 
A multivariate generalization of classical order statistics for random samples from a continuous multivariate distribution was defined by Corley [18]. Guilbaud [19] expressed the probability of the functions of innid random vectors as a linear combination of probabilities of the functions of independent and identically distributed (iid) random vectors and thus also for order statistics of random variables. Expressions for generalized joint densities of order statistics of iid random variables in terms of Radon-Nikodym derivatives with respect to product measures based on $d f$ were derived by Goldie and Maller [20].

Several identities and recurrence relations for $p d f$ and $d f$ of order statistics of $i i d$ random variables were established by numerous authors including Arnold et al. [21], Balasubramanian and Beg [22], David [23], and Reiss [24]. Furthermore, Arnold et al. [21], David [23], Gan and Bain [25], and Khatri [26] obtained the probability function $(p f)$ and $d f$ of order statistics of iid random variables from a discrete parent. Balakrishnan [27] showed that several relations and identities that have been derived for order statistics from continuous distributions also hold for the discrete case. In 1986, Nagaraja [28] explored the behavior of higher order conditional probabilities of order statistics in an attempt to understand the structure of discrete order statistics. Later, Nagaraja [29] considered some results on order statistics of a random sample taken from a discrete population.

In general, the distribution theory for order statistics is complex when the parent distribution is discrete. In this study, the joint distributions of order statistics of innid discrete random variables are expressed in the form of an integral. As far as we know, these approaches have not been considered in the framework of order statistics from innid discrete random variables.

From now on, the subscripts and superscripts are defined at first usage, and these definitions will be valid unless they are redefined.

If $\mathrm{a}_{1}, \mathrm{a}_{2}, \ldots$ are column vectors, then $\left[\begin{array}{cc}\mathrm{a}_{1} & \mathrm{a}_{2} \\ m_{1} & m_{2}\end{array}\right]$ ] will denote the matrix obtained by taking $m_{1}$ copies of $\mathrm{a}_{1}, m_{2}$ copies of $\mathrm{a}_{2}$ and so on. per A denotes the permanent of a square matrix A; the permanent is defined just like the determinant, except that all signs in the expansion are positive.

Let $X_{1}, X_{2}, \ldots, X_{n}$ be innid discrete random variables and $X_{1: n} \leq X_{2: n} \leq \cdots \leq X_{n: n}$ be the order statistics obtained by arranging the $n X_{i}$ 's in the increasing order of magnitude. Let $F_{i}$ and $f_{i}$ be $d f$ and $p f$ of $X_{i}(i=1,2, \ldots, n)$, respectively.

The $d f$ and $p f$ of $X_{r_{1}: n}, X_{r_{2}: n}, \ldots, X_{r_{p}: n}, 1 \leq r_{1}<r_{2}<\cdots<r_{n} \leq n(p=1,2, \ldots, n)$ will be given. For notational convenience, we write $\sum_{z_{1}, z_{2}, \ldots, z_{p}}, \sum_{m_{p}, k_{p}, \ldots, m_{1}, k_{1}}, \int$ and $\int_{\mathrm{V}}$ instead of $\sum_{z_{1}=0}^{x_{1}} \sum_{z_{2}=z_{1}}^{x_{2}} \sum_{z_{3}=z_{2}}^{x_{3}} \cdots \sum_{z_{p}=z_{p-1}}^{x_{p}}, \sum_{m_{p}=0}^{n-r_{p}} \sum_{k_{p}=0}^{r_{p}-1-r_{p-1}} \cdots \sum_{m_{2}=0}^{r_{3}-1-r_{2}} \sum_{k_{2}=0}^{r_{2}-1-r_{1}} \sum_{m_{1}=0}^{r_{2}-1-r_{1}} \sum_{k_{1}=0}^{r_{1}-1}$,

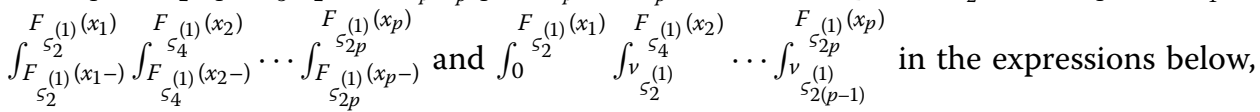
respectively $\left(x_{i}=0,1,2, \ldots\right)\left(z_{0}=0\right)$.

\section{Theorems for distribution and probability functions}

In this section, the theorems related to $p f$ and $d f$ of $X_{r_{1}: n}, X_{r_{2}: n}, \ldots, X_{r_{p}: n}$ will be given. We will now express the following theorem for the joint $p f$ of order statistics of innid discrete random variables. 


\section{Theorem 1}

$$
\begin{aligned}
& f_{r_{1}, r_{2}, \ldots, r_{p}: n}\left(x_{1}, x_{2}, \ldots, x_{p}\right) \\
& =\sum_{m_{p}, k_{p}, \ldots, m_{1}, k_{1}} \sum_{n_{s_{1}}, n_{s_{2}}, \ldots, n_{s_{2}}}\left(\prod_{w=1}^{p+1} \prod_{i_{2 w-1}=1}^{r_{w}-1-k_{w}-m_{w-1}-r_{w-1}}\left[F_{s_{2 w-1}\left(i_{2 w-1}\right)}\left(x_{w}-\right)-F_{s_{2 w-1}}\left(_{i_{2} i_{2}-1}\left(x_{w-1}\right)\right]\right)\right. \\
& \quad \times \prod_{w=1}^{p} \prod_{i_{2 \mathrm{w}}=1}^{k_{w}+1+m_{w}} f_{s_{2 \mathrm{w}}}\left(x_{w}\right),
\end{aligned}
$$

where $x_{1}<x_{2}<\cdots<x_{p}, \sum_{n_{s_{1}}, n_{s_{2}}, \ldots, n_{s_{2}}}$ denotes the sum over $\bigcup_{\ell=1}^{2 p} s_{\ell}$ for which $s_{v} \cap s_{\vartheta}=\phi$ for $v \neq \vartheta, S=\bigcup_{\ell=1}^{2 p+1} s_{\ell}, S=\{1,2, \ldots, n\}, r_{0}=0, r_{p+1}=n+1, m_{0}=0, k_{p+1}=0, m_{w-1}+k_{w} \leq$ $r_{w}-r_{w-1}-1, F_{s_{1}^{\left(i_{1}\right)}}\left(x_{0}\right)=0, F_{s_{2 p+1}}\left(i_{2 p+1}-\right)=1, F_{i}\left(x_{w}-\right)=P\left(X_{i}<x_{w}\right)(w=1,2, \ldots, p+1), n_{s_{\ell}}$ is the cardinality of $s_{\ell}$ and

$$
s_{\ell}= \begin{cases}\left\{s_{\ell}^{(1)}, s_{\ell}^{(2)}, \ldots, s_{\ell}^{\left(k_{\ell}+1+m_{\ell}\right)} \frac{1}{2}\right\}, & \text { if } \ell \text { even }, \\ \left\{s_{\ell}^{(1)}, s_{\ell}^{(2)}, \ldots, s_{\ell}{ }^{\left.\frac{\left(r_{+1}\right.}{2}-1-k_{\frac{\ell+1}{2}}-m_{\frac{\ell-1}{2}}-r_{\frac{\ell-1}{2}}\right)}\right\}, & \text { if } \ell \text { odd } .\end{cases}
$$

Proof Consider the event $\left\{X_{r_{1}: n}=x_{1}, X_{r_{2}: n}=x_{2}, \ldots, X_{r_{p}: n}=x_{p}\right\}$.

The above event can be realized in mutually exclusive ways as follows: $r_{1}-1-k_{1}$ observations are less than $x_{1}, k_{w}+1+m_{w}(w=1,2, \ldots, p)$ observations are equal to $x_{w}$, $r_{\xi}-1-k_{\xi}-m_{\xi-1}-r_{\xi-1}(\xi=2,3, \ldots, p)$ observations are in interval $\left(x_{\xi-1}, x_{\xi}\right)$ and $n-m_{p}-r_{p}$ observations exceed $x_{p}$. The probability function of the above event can be written as

$$
f_{r_{1}, r_{2}, \ldots, r_{p}: n}\left(x_{1}, x_{2}, \ldots, x_{p}\right)=P\left\{X_{r_{1}: n}=x_{1}, X_{r_{2}: n}=x_{2}, \ldots, X_{r_{p}: n}=x_{p}\right\}
$$

Identity (2) can be expressed as

$$
\begin{aligned}
& f_{r_{1}, r_{2}, \ldots, r_{p}: n}\left(x_{1}, x_{2}, \ldots, x_{p}\right)
\end{aligned}
$$

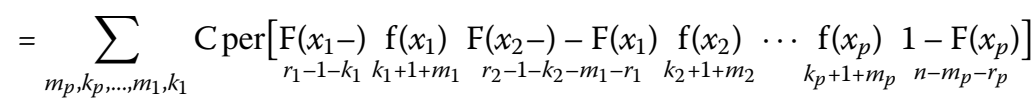

$$
\begin{aligned}
& =\sum_{m_{p}, k_{p}, \ldots, m_{1}, k_{1}} \mathrm{C} \sum_{n_{s_{1}}, n_{s_{2}}, \ldots, n_{s_{2 p}}} \operatorname{per}\left[\mathrm{F}\left(x_{1}-\right)\right]\left[s_{1} / \cdot\right) \\
& \times \operatorname{per}\left[\underset{k_{1}+1+m_{1}}{\mathrm{f}}\left(x_{1}\right)\right]\left[s_{2} / \cdot\right) \operatorname{per}\left[\underset{r_{2}-1-k_{2}-m_{1}-r_{1}}{\left.\mathrm{~F}\left(x_{2}-\right)-\mathrm{F}\left(x_{1}\right)\right]\left[s_{3} / \cdot\right)}\right.
\end{aligned}
$$

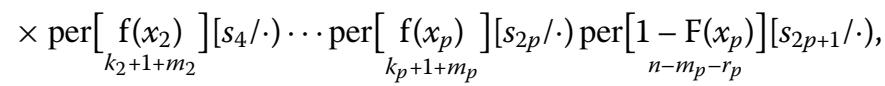

where $\mathrm{C}=\left(\prod_{w=1}^{p+1}\left[\left(r_{w}-1-k_{w}-m_{w-1}-r_{w-1}\right) !\right]^{-1}\right) \prod_{w=1}^{p}\left[\left(k_{w}+1+m_{w}\right) !\right]^{-1}, \mathrm{~F}\left(x_{w}\right)=\left(F_{1}\left(x_{w}\right)\right.$, $\left.F_{2}\left(x_{w}\right), \ldots, F_{n}\left(x_{w}\right)\right)^{\prime}$ and $\mathrm{f}\left(x_{w}\right)=\left(f_{1}\left(x_{w}\right), f_{2}\left(x_{w}\right), \ldots, f_{n}\left(x_{w}\right)\right)^{\prime}$ are column vectors. $\mathrm{A}\left[s_{\ell} / \cdot\right)$ is the matrix obtained from A by taking rows whose indices are in $s_{\ell}$. Using the expansion of the permanent in the above identity, we get (1).

Identity (1) can also be written in the form of an integral as follows. 
Theorem 2

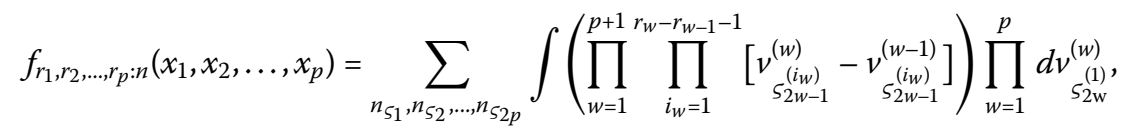

where $x_{1}<x_{2}<\cdots<x_{p}, \sum_{n_{51}, n_{52}, \ldots, n_{52 p}}$ denotes the sum over $\bigcup_{\ell=1}^{2 p} \varsigma_{\ell}$ for which $\varsigma_{v} \cap \varsigma_{\vartheta}=\phi$ for $v \neq \vartheta, S=\bigcup_{\ell=1}^{2 p+1} \varsigma_{\ell}, v_{\varsigma_{2 w-1}^{(t)}}^{\left(i_{w}\right)}=\left[v_{\varsigma_{2 t}^{(1)}}^{(t)}-F_{\varsigma_{2 t}^{(1)}}\left(x_{t}-\right)\right] \frac{f_{2 w-1}^{\left(i_{w}\right)}\left(x_{t}\right)}{f_{s_{2 t}(1)}^{(1)}\left(x_{t}\right)}+F_{\varsigma_{2 w-1}^{\left(i_{w}\right)}}\left(x_{t}-\right), v_{\varsigma_{1}^{\left(i_{1}\right)}}^{(0)}=0, v_{\varsigma_{2 p+1}^{\left(i_{p+1}\right)}}^{(p+1)}=1$ and

$$
\varsigma_{\ell}= \begin{cases}\left\{\varsigma_{\ell}^{(1)}\right\} & \text { if } \ell \text { even } \\ \left\{\varsigma_{\ell}^{(1)}, \varsigma_{\ell}^{(2)}, \ldots, \varsigma_{\ell}^{\left(r_{\frac{\ell+1}{2}}-r_{\frac{\ell-1}{2}}^{-1)}\right.}\right\}, & \text { if } \ell \text { odd }\end{cases}
$$

Proof Consider the identity

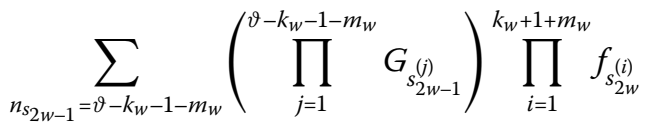

$$
\begin{aligned}
& =\frac{k_{w} ! m_{w} !}{\left(k_{w}+1+m_{w}\right) !} \sum_{\substack{n_{\tau_{4 w-3}}=\vartheta-k_{w}-1-m_{w} \\
n_{\tau_{4 w-2}}=k_{w} \\
n_{\tau_{4 w-1}}=1}}\left(\prod_{i_{1}=1}^{\vartheta-k_{w}-1-m_{w}} G_{\tau_{4 w-3}^{\left(i_{1}\right)}}\right)\left(\prod_{i_{2}=1}^{k_{w}} f_{\tau_{4 w-2}\left(i_{2}\right)}\right) f_{\tau_{4 w-1}^{(1)}} \prod_{i_{3}=1}^{m_{w}} f_{\tau_{4 w}}
\end{aligned}
$$

and using (4) in (1), it can be written as

$$
\begin{aligned}
& f_{r_{1}, r_{2}, \ldots, r_{p}: n}\left(x_{1}, x_{2}, \ldots, x_{p}\right) \\
& =\sum_{m_{p}, k_{p}, \ldots, m_{1}, k_{1}}\left(\prod_{w=1}^{p} \frac{k_{w} ! m_{w} !}{\left(k_{w}+1+m_{w}\right) !}\right) \sum_{n_{\tau_{1}}, n_{\tau_{2}}, \ldots, n_{\tau_{4} p}}\left[\prod_{w=1}^{p+1}\left(\prod_{i_{3(w-1)}=1}^{m_{w-1}} f_{\tau_{4(w-1)}\left(i_{3(w-1)}\right)}\left(x_{w-1}\right)\right)\right. \\
& \quad \times\left(\prod_{i_{3 w-2}=1}^{r_{w}-1-k_{w}-m_{w-1}-r_{w-1}}\left[F_{\tau_{4 w-3}^{\left(i_{3 w-2}\right)}}\left(x_{w}-\right)-F_{\tau_{4 w-3}^{\left(i_{3 w-2}\right)}}\left(x_{w-1}\right)\right]\right) \\
& \left.\quad \times \prod_{i_{3 w-1}=1}^{k_{w}} f_{\tau_{4 w-2}^{\left(i_{3 w-1}\right)}}\left(x_{w}\right)\right] \prod_{w=1}^{p} f_{\tau_{4 w-1}^{(1)}}\left(x_{w}\right)
\end{aligned}
$$

where $\sum_{n_{\tau_{1}}, n_{\tau_{2}}, \ldots, n_{\tau_{4 p}}}$ denotes the sum over $\bigcup_{l=1}^{4 p} \tau_{l}$ for which $\tau_{v} \cap \tau_{\vartheta}=\phi$ for $v \neq \vartheta, S=$ $\bigcup_{l=1}^{4 p+1} \tau_{l}$

$$
\begin{aligned}
& s_{2 w}=\tau_{4 w-2} \cup \tau_{4 w-1} \cup \tau_{4 w}, \quad s_{2 w-1}=\tau_{4 w-3} \quad \text { and } \\
& \tau_{l}= \begin{cases}\left\{\tau_{l}^{(1)}, \tau_{l}^{(2)}, \ldots, \tau_{l}^{\left(m_{l}\right)}{ }^{\frac{(2)}{4}}\right\}, & \text { if } l \equiv 0(\bmod 4), \\
\left\{\tau_{l}^{(1)}, \tau_{l}^{(2)}, \ldots, \tau_{l}^{\left.{ }^{\left(\frac{l+3}{4}\right.}-1-m_{\frac{l-1}{4}}-k_{\frac{l+3}{4}}-r_{\frac{l-1}{4}}\right)}\right\}, & \text { if } l \equiv 1(\bmod 4), \\
\left\{\tau_{l}^{(1)}, \tau_{l}^{(2)}, \ldots, \tau_{l}{ }^{\left(k_{l+2}\right)}\right\}, & \text { if } l \equiv 2(\bmod 4), \\
\left\{\tau_{l}^{(1)}\right\}, & \text { if } l \equiv 3(\bmod 4) .\end{cases}
\end{aligned}
$$


The above identity can be written as

$$
\begin{aligned}
& f_{r_{1}, r_{2}, \ldots, r_{p}: n}\left(x_{1}, x_{2}, \ldots, x_{p}\right) \\
& =\sum_{m_{p}, k_{p}, \ldots, m_{1}, k_{1}}\left(\prod_{w=1}^{p} \frac{k_{w} ! m_{w} !}{\left(k_{w}+1+m_{w}\right) !}\right) \\
& \times \sum_{n_{\tau_{1}}, n_{\tau_{2}}, \ldots, n_{\tau_{4 p}}}\left(\prod_{w=1}^{p} \frac{\left(k_{w}+1+m_{w}\right) !}{k_{w} ! m_{w} !}\right) \\
& \times\left[\int_{0}^{1} \int_{0}^{1} \cdots \int_{0}^{1} y_{1}^{k_{1}}\left(1-y_{1}\right)^{m_{1}} y_{2}^{k_{2}}\left(1-y_{2}\right)^{m_{2}} \cdots y_{p}^{k_{p}}\left(1-y_{p}\right)^{m_{p}} d y_{1} d y_{2} \cdots d y_{p}\right] \\
& \times\left[\prod_{w=1}^{p+1}\left(\prod_{i_{3(w-1)}=1}^{m_{w-1}} f_{\tau_{4(w-1)}\left(i_{3(w-1)}\right)}\left(x_{w-1}\right)\right)\right. \\
& \times\left(\prod_{i_{3 w-2}=1}^{r_{w}-1-k_{w}-m_{w-1}-r_{w-1}}\left[F_{\tau_{4 w-3}^{\left(i_{3 w-2}\right)}}\left(x_{w}-\right)-F_{\tau_{4 w-3}^{\left(i_{3 w-2}\right)}}\left(x_{w-1}\right)\right]\right) \\
& \left.\times \prod_{i_{3 w-1}=1}^{k_{w}} f_{\tau_{4 w-2}^{\left(i_{3 w-1}\right)}}\left(x_{w}\right)\right] \prod_{w=1}^{p} f_{\tau_{4 \mathrm{w}-1}^{(1)}}\left(x_{w}\right) .
\end{aligned}
$$

The following expression can be written from the above identity

$$
\begin{aligned}
f_{r_{1}, r_{2}, \ldots, r_{p}: n}\left(x_{1}, x_{2}, \ldots, x_{p}\right) & \\
= & \sum_{m_{p}, k_{p}, \ldots, m_{1}, k_{1}} \sum_{n_{\tau_{1}}, n_{\tau_{2}, \ldots, \ldots, n_{\tau_{4 p}}}} \int_{0}^{1} \int_{0}^{1} \cdots \int_{0}^{1}\left[\prod_{w=1}^{p+1}\left(\prod_{i_{3(w-1)}=1}^{m_{w-1}}\left(1-y_{w-1}\right) f_{\tau_{4(w-1)}\left(i_{3(w-1)}\right.}\left(x_{w-1}\right)\right)\right. \\
& \times\left(\prod_{i_{3 w-2}=1}^{r_{w}-1-k_{w}-m_{w-1}-r_{w-1}}\left[F_{\tau_{4 w-3}^{\left(i_{3 w-2}\right)}}\left(x_{w}-\right)-F_{\tau_{4 w-3}^{\left(i_{3 w-2}\right)}}\left(x_{w-1}\right)\right]\right) \\
& \left.\times \prod_{i_{3 w-1}=1}^{k_{w}} y_{w} f_{\tau_{4 w-2}}^{\left(i_{3 w-1}\right)}\left(x_{w}\right)\right] \prod_{w=1}^{p} f_{\tau_{4 w-1}^{(1)}}\left(x_{w}\right) d y_{w}
\end{aligned}
$$

and here, if $v_{\tau_{l}}^{\left(\left(_{j}\right)\right.}=y_{w} f_{\tau_{l}}\left(i_{j}\right)\left(x_{w}\right)+F_{\tau_{l}}^{\left(i_{j}\right)}\left(x_{w}-\right)$, the following identity is obtained

$$
\begin{aligned}
& f_{r_{1}, r_{2}, \ldots, r_{p}: n}\left(x_{1}, x_{2}, \ldots, x_{p}\right)
\end{aligned}
$$

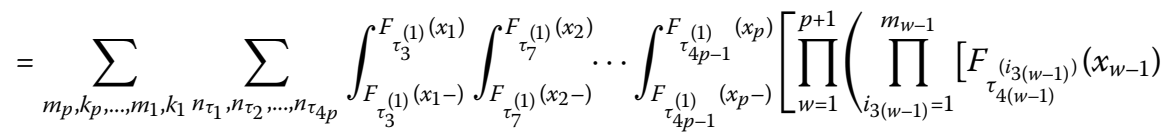

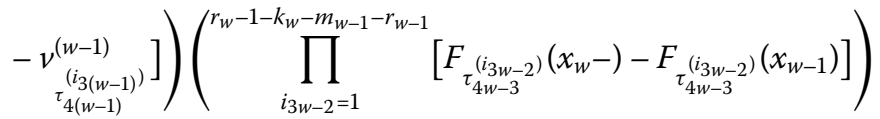

$$
\begin{aligned}
& \left.\times \prod_{i_{3 w-1}=1}^{k_{w}}\left[v_{\tau_{4 w-2}^{(w)}}^{\left(i_{3 w-1}\right)}-F_{\tau_{4 w-2}}^{\left(i_{3 w-1}\right)}\left(x_{w}-\right)\right]\right] \prod_{w=1}^{p} d v_{\tau_{4 w-1}^{(w)}}^{(1)}
\end{aligned}
$$


By considering

$$
\begin{aligned}
& \sum_{\xi=0}^{l} \sum_{\zeta=0}^{l} \sum_{\substack{n_{\tau_{4(w-1)}} \zeta \\
n_{\tau_{4 w-3}}=l-\xi-\zeta}}\left(\prod_{i_{3(w-1)}=1}^{\zeta} g_{\tau_{4(w-1)}^{\left(i_{3(w-1)}\right)}}\right)\left(\prod_{i_{3 w-2}=1}^{l-\xi-\zeta} f_{\tau_{4 w-3}^{\left(i_{3 w-2}\right)}}\right) \prod_{i_{3 w-1}=1}^{\xi} h_{\tau_{4 w-2}^{\left(i_{3 w-1}\right)}} \\
& \quad=\prod_{i_{w}=1}^{l}\left[g_{S_{2 w-1}^{\left(i_{w}\right)}}+f_{S_{2 w-1}^{\left(i_{w}\right)}}+h_{\left.S_{2 w-1}^{\left(i_{w}\right)}\right]}\right],
\end{aligned}
$$

where $\xi+\zeta \leq l$, and using (6) for each $m_{w-1}$ and $k_{w}$ in (5), we get

$$
\begin{aligned}
& f_{r_{1}, r_{2}, \ldots, r_{p}: n}\left(x_{1}, x_{2}, \ldots, x_{p}\right) \\
& =\sum_{n_{S 1}, n_{S 2}, \ldots, n_{S 2 p}} \int\left(\prod _ { w = 1 } ^ { p + 1 } \prod _ { i _ { w } = 1 } ^ { r _ { w } - r _ { w - 1 } - 1 } \left[F_{\varsigma_{2 w-1}^{\left(i_{w}\right)}}\left(x_{w-1}\right)-v_{\varsigma_{2 w-1}^{\left(i_{w}\right)}}^{(w-1)}+F_{\varsigma_{2 w-1}^{\left(i_{w}\right)}}\left(x_{w}-\right)\right.\right. \\
& \left.\left.\quad-F_{\varsigma_{2 w-1}^{\left(i_{w}\right)}}\left(x_{w-1}\right)+v_{\varsigma_{2 w-1}^{(w)}\left(i_{w}\right)}^{(w)}-F_{\varsigma_{2 w-1}^{\left(i_{w}\right)}}\left(x_{w}-\right)\right]\right) \prod_{w=1}^{p} d v_{s_{2 w}^{(1)}}^{(w)},
\end{aligned}
$$

where $\varsigma_{2 w-1}=\tau_{4(w-1)} \cup \tau_{4 w-3} \cup \tau_{4 w-2}$ and $\varsigma_{2 w}=\tau_{4 w-1}$. This completes the proof of the theorem.

We have the following special cases obtained from (3). Consider by taking $p=2, n=$ $3, r_{1}=1, r_{2}=2$ and $v_{\varsigma_{5}^{(1)}}^{(2)}=\left[v_{\varsigma_{4}^{(1)}}^{(2)}-F_{\varsigma_{4}^{(1)}}\left(x_{2}-\right)\right] \frac{f_{5}^{(1)}\left(x_{2}\right)}{f_{s_{4}}^{(1)}\left(x_{2}\right)}+F_{\varsigma_{5}^{(1)}}\left(x_{2}-\right)$, the following identity is obtained

$$
\begin{aligned}
& f_{1,2: 3}\left(x_{1}, x_{2}\right)=\sum_{n_{S 2}, n_{S 4}} \int_{F_{\varsigma_{2}}^{(1)}\left(x_{1}-\right)}^{F s_{2}^{(1)}\left(x_{1}\right)}\left(\int_{F_{\varsigma_{4}}^{(1)}\left(x_{2}-\right)}^{F_{s_{4}^{(1)}}^{\left(x_{2}\right)}}\left(1-v_{\varsigma_{5}^{(1)}}^{(2)}\right) d v_{\varsigma_{4}^{(1)}}^{(2)}\right) d v_{\varsigma_{2}^{(1)}}^{(1)} \\
& =\sum_{n_{52}, n_{54}} f_{\varsigma_{2}^{(1)}}\left(x_{1}\right)\left\{f_{\varsigma_{4}^{(1)}}\left(x_{2}\right)+\frac{1}{2} f_{\varsigma_{5}^{(1)}}\left(x_{2}\right) F_{\varsigma_{4}^{(1)}}\left(x_{2}-\right)\right. \\
& \left.-\frac{1}{2} f_{\varsigma_{5}^{(1)}}\left(x_{2}\right) F_{\varsigma_{4}^{(1)}}\left(x_{2}\right)-f_{\varsigma_{4}^{(1)}}\left(x_{2}\right) F_{\varsigma_{5}^{(1)}}\left(x_{2}-\right)\right\} \\
& =f_{1}\left(x_{1}\right)\left\{f_{2}\left(x_{2}\right)+\frac{1}{2} f_{3}\left(x_{2}\right) F_{2}\left(x_{2}-\right)-\frac{1}{2} f_{3}\left(x_{2}\right) F_{2}\left(x_{2}\right)-f_{2}\left(x_{2}\right) F_{3}\left(x_{2}-\right)\right\} \\
& +f_{1}\left(x_{1}\right)\left\{f_{3}\left(x_{2}\right)+\frac{1}{2} f_{2}\left(x_{2}\right) F_{3}\left(x_{2}-\right)-\frac{1}{2} f_{2}\left(x_{2}\right) F_{3}\left(x_{2}\right)-f_{3}\left(x_{2}\right) F_{2}\left(x_{2}-\right)\right\} \\
& +f_{2}\left(x_{1}\right)\left\{f_{3}\left(x_{2}\right)+\frac{1}{2} f_{1}\left(x_{2}\right) F_{3}\left(x_{2}-\right)-\frac{1}{2} f_{1}\left(x_{2}\right) F_{3}\left(x_{2}\right)-f_{3}\left(x_{2}\right) F_{1}\left(x_{2}-\right)\right\} \\
& +f_{2}\left(x_{1}\right)\left\{f_{1}\left(x_{2}\right)+\frac{1}{2} f_{3}\left(x_{2}\right) F_{1}\left(x_{2}-\right)-\frac{1}{2} f_{3}\left(x_{2}\right) F_{1}\left(x_{2}\right)-f_{1}\left(x_{2}\right) F_{3}\left(x_{2}-\right)\right\} \\
& +f_{3}\left(x_{1}\right)\left\{f_{1}\left(x_{2}\right)+\frac{1}{2} f_{2}\left(x_{2}\right) F_{1}\left(x_{2}-\right)-\frac{1}{2} f_{2}\left(x_{2}\right) F_{1}\left(x_{2}\right)-f_{1}\left(x_{2}\right) F_{2}\left(x_{2}-\right)\right\} \\
& +f_{3}\left(x_{1}\right)\left\{f_{2}\left(x_{2}\right)+\frac{1}{2} f_{1}\left(x_{2}\right) F_{2}\left(x_{2}-\right)-\frac{1}{2} f_{1}\left(x_{2}\right) F_{2}\left(x_{2}\right)-f_{2}\left(x_{2}\right) F_{1}\left(x_{2}-\right)\right\} \text {. }
\end{aligned}
$$


Moreover, the above identity in the iid case can be expressed by

$$
f_{1,2: 3}\left(x_{1}, x_{2}\right)=6 f\left(x_{1}\right) f\left(x_{2}\right)-6 f\left(x_{1}\right) f\left(x_{2}\right) F\left(x_{2}\right)+3 f\left(x_{1}\right) f^{2}\left(x_{2}\right) .
$$

This result is obtained if $i=1, j=2$, and $n=3$ in equation (6) in Khatri [26].

If $x_{1}=x_{2}=\cdots=x_{p}=x$, it should be written as $\iint \cdots \int$ instead of $\int$ in (3), where $\iint \cdots \int$ is to be carried out over the region: $F_{s_{2}^{(1)}}\left(x_{1}-\right) \leq v_{\varsigma_{2}^{(1)}}^{(1)} \leq v_{\varsigma_{4}^{(1)}}^{(2)} \leq \cdots \leq v_{\varsigma_{2 p}^{(1)}}^{(p)} \leq$ $F_{\varsigma_{2 \mathrm{p}}^{(1)}}\left(x_{p^{-}}-\right), F_{\varsigma_{2}^{(1)}}\left(x_{1}-\right) \leq v_{\varsigma_{2}^{(1)}}^{(1)} \leq F_{\varsigma_{2}^{(1)}}\left(x_{1}\right), F_{\varsigma_{4}^{(1)}}\left(x_{2}-\right) \leq v_{\varsigma_{4}^{(1)}}^{(2)} \leq F_{\varsigma_{4}^{(1)}}\left(x_{2}\right), \ldots, F_{\varsigma_{2 \mathrm{p}}^{(1)}}\left(x_{p}-\right) \leq v_{\varsigma_{2 \mathrm{p}}^{(1)}}^{(p)} \leq$ $F_{\varsigma_{2 \mathrm{p}}^{(1)}}\left(x_{p}\right)$.

Further on considering $p=2, n=3, r_{1}=1, r_{2}=2$, and $v_{\varsigma_{5}^{(1)}}^{(2)}=\left[v_{\varsigma_{4}^{(1)}}^{(2)}-F_{\varsigma_{4}^{(1)}}\left(x_{2}-\right)\right] \frac{f_{5}^{(1)}\left(x_{2}\right)}{f_{\varsigma_{4}(1)}^{\left(x_{2}\right)}}+$ $F_{\varsigma_{5}^{(1)}}\left(x_{2}-\right)$ in (3), if $X_{1}, X_{2}, X_{3}$ are innid discrete random variables and for $x_{1}=x_{2}=x$, then

$$
\begin{aligned}
& f_{1,2: 3}(x, x) \\
& =\sum_{n_{52}, n_{\zeta 4}} \int_{F_{\varsigma_{2}}^{(1)}(x-)}^{F_{s_{2}^{(1)}}^{(x)}}\left(\int_{v_{\varsigma_{2}}^{(1)}}^{F_{\varsigma_{4}^{(1)}}^{(x)}}\left(1-v_{\varsigma_{5}^{(1)}}^{(2)}\right) d v_{\varsigma_{4}^{(1)}}^{(2)}\right) d v_{\varsigma_{2}^{(1)}}^{(1)} \\
& =\sum_{n_{\zeta 2}, n_{\zeta 4}}\left\{F_{\varsigma_{4}^{(1)}}(x) f_{\varsigma_{2}^{(1)}}(x)-\frac{1}{2}\left[F_{\varsigma_{2}^{(1)}}(x)+F_{\varsigma_{2}^{(1)}}(x-)\right] f_{\varsigma_{2}^{(1)}}(x)-\frac{1}{2} F_{\varsigma_{4}^{(1)}}^{2}(x) f_{\varsigma_{2}^{(1)}}(x) \frac{f_{\varsigma_{5}^{(1)}}(x)}{f_{\zeta_{4}(1)}(x)}\right. \\
& +\frac{1}{6}\left[F_{\varsigma_{2}^{(1)}}^{3}(x)-F_{\varsigma_{2}^{(1)}}^{3}(x-)\right] \frac{f_{\varsigma_{5}^{(1)}}(x)}{f_{\varsigma_{4}^{(1)}}(x)}+F_{\varsigma_{4}^{(1)}}(x) F_{\varsigma_{4}^{(1)}}(x-) \frac{f_{\varsigma_{2}^{(1)}}(x) f_{\varsigma_{5}^{(1)}}(x)}{f_{\varsigma_{4}^{(1)}}(x)} \\
& -\frac{1}{2}\left[F_{\varsigma_{2}^{(1)}}(x)+F_{\varsigma_{2}^{(1)}}(x-)\right] f_{\varsigma_{2}^{(1)}}(x) F_{\varsigma_{4}^{(1)}}(x-) \frac{f_{\zeta_{5}^{(1)}}(x)}{f_{\varsigma_{4}^{(1)}}(x)}-F_{\varsigma_{4}^{(1)}}(x) F_{\varsigma_{5}^{(1)}}(x-) f_{\varsigma_{2}^{(1)}}(x) \\
& \left.+\frac{1}{2}\left[F_{\varsigma_{2}^{(1)}}(x)+F_{\varsigma_{2}^{(1)}}(x-)\right] f_{\varsigma_{2}^{(1)}}(x) F_{\varsigma_{5}^{(1)}}(x-)\right\} \\
& =\left\{F_{2}(x) f_{1}(x)-\frac{1}{2}\left[F_{1}(x)+F_{1}(x-)\right] f_{1}(x)-\frac{1}{2} F_{2}^{2}(x) f_{1}(x) \frac{f_{3}(x)}{f_{2}(x)}+\frac{1}{6}\left[F_{1}^{3}(x)-F_{1}^{3}(x-)\right] \frac{f_{3}(x)}{f_{2}(x)}\right. \\
& +F_{2}(x) F_{2}(x-) \frac{f_{1}(x) f_{3}(x)}{f_{2}(x)}-\frac{1}{2}\left[F_{1}(x)+F_{1}(x-)\right] f_{1}(x) F_{2}(x-) \frac{f_{3}(x)}{f_{2}(x)}-F_{2}(x) F_{3}(x-) f_{1}(x) \\
& \left.+\frac{1}{2}\left[F_{1}(x)+F_{1}(x-)\right] f_{1}(x) F_{3}(x-)\right\} \\
& +\left\{F_{3}(x) f_{1}(x)-\frac{1}{2}\left[F_{1}(x)+F_{1}(x-)\right] f_{1}(x)-\frac{1}{2} F_{3}^{2}(x) f_{1}(x) \frac{f_{2}(x)}{f_{3}(x)}\right. \\
& +\frac{1}{6}\left[F_{1}^{3}(x)-F_{1}^{3}(x-)\right] \frac{f_{2}(x)}{f_{3}(x)}+F_{3}(x) F_{3}(x-) \frac{f_{1}(x) f_{2}(x)}{f_{3}(x)} \\
& -\frac{1}{2}\left[F_{1}(x)+F_{1}(x-)\right] f_{1}(x) F_{3}(x-) \frac{f_{2}(x)}{f_{3}(x)}-F_{3}(x) F_{2}(x-) f_{1}(x) \\
& \left.+\frac{1}{2}\left[F_{1}(x)+F_{1}(x-)\right] f_{1}(x) F_{2}(x-)\right\} \\
& +\left\{F_{1}(x) f_{2}(x)-\frac{1}{2}\left[F_{2}(x)+F_{2}(x-)\right] f_{2}(x)-\frac{1}{2} F_{1}^{2}(x) f_{2}(x) \frac{f_{3}(x)}{f_{1}(x)}\right.
\end{aligned}
$$




$$
\begin{aligned}
& +\frac{1}{6}\left[F_{2}^{3}(x)-F_{2}^{3}(x-)\right] \frac{f_{3}(x)}{f_{1}(x)}+F_{1}(x) F_{1}(x-) \frac{f_{2}(x) f_{3}(x)}{f_{1}(x)} \\
& -\frac{1}{2}\left[F_{2}(x)+F_{2}(x-)\right] f_{2}(x) F_{1}(x-) \frac{f_{3}(x)}{f_{1}(x)}-F_{1}(x) F_{3}(x-) f_{2}(x) \\
& \left.+\frac{1}{2}\left[F_{2}(x)+F_{2}(x-)\right] f_{2}(x) F_{3}(x-)\right\} \\
& +\left\{F_{3}(x) f_{2}(x)-\frac{1}{2}\left[F_{2}(x)+F_{2}(x-)\right] f_{2}(x)-\frac{1}{2} F_{3}^{2}(x) f_{2}(x) \frac{f_{1}(x)}{f_{3}(x)}\right. \\
& +\frac{1}{6}\left[F_{2}^{3}(x)-F_{2}^{3}(x-)\right] \frac{f_{1}(x)}{f_{3}(x)}+F_{3}(x) F_{3}(x-) \frac{f_{2}(x) f_{1}(x)}{f_{3}(x)} \\
& -\frac{1}{2}\left[F_{2}(x)+F_{2}(x-)\right] f_{2}(x) F_{3}(x-) \frac{f_{1}(x)}{f_{3}(x)}-F_{3}(x) F_{1}(x-) f_{2}(x) \\
& \left.+\frac{1}{2}\left[F_{2}(x)+F_{2}(x-)\right] f_{2}(x) F_{1}(x-)\right\} \\
& +\left\{F_{2}(x) f_{3}(x)-\frac{1}{2}\left[F_{3}(x)+F_{3}(x-)\right] f_{3}(x)-\frac{1}{2} F_{2}^{2}(x) f_{3}(x) \frac{f_{1}(x)}{f_{2}(x)}\right. \\
& +\frac{1}{6}\left[F_{3}^{3}(x)-F_{3}^{3}(x-)\right] \frac{f_{1}(x)}{f_{2}(x)}+F_{2}(x) F_{2}(x-) \frac{f_{3}(x) f_{1}(x)}{f_{2}(x)} \\
& -\frac{1}{2}\left[F_{3}(x)+F_{3}(x-)\right] f_{3}(x) F_{2}(x-) \frac{f_{1}(x)}{f_{2}(x)}-F_{2}(x) F_{1}(x-) f_{3}(x) \\
& \left.+\frac{1}{2}\left[F_{3}(x)+F_{3}(x-)\right] f_{3}(x) F_{1}(x-)\right\} \\
& +\left\{F_{1}(x) f_{3}(x)-\frac{1}{2}\left[F_{3}(x)+F_{3}(x-)\right] f_{3}(x)-\frac{1}{2} F_{1}^{2}(x) f_{3}(x) \frac{f_{2}(x)}{f_{1}(x)}\right. \\
& +\frac{1}{6}\left[F_{3}^{3}(x)-F_{3}^{3}(x-)\right] \frac{f_{2}(x)}{f_{1}(x)}+F_{1}(x) F_{1}(x-) \frac{f_{3}(x) f_{2}(x)}{f_{1}(x)} \\
& -\frac{1}{2}\left[F_{3}(x)+F_{3}(x-)\right] f_{3}(x) F_{1}(x-) \frac{f_{2}(x)}{f_{1}(x)}-F_{1}(x) F_{2}(x-) f_{3}(x) \\
& \left.+\frac{1}{2}\left[F_{3}(x)+F_{3}(x-)\right] f_{3}(x) F_{2}(x-)\right\} \text {. }
\end{aligned}
$$

Moreover, the above identity in the iid case can be expressed by

$$
\begin{aligned}
= & 6 F(x) f(x)-3[F(x)+F(x-)] f(x)-3 F^{2}(x) f(x)+\left[F^{3}(x)-F^{3}(x-)\right]+6 F(x) F(x-) f(x) \\
& -3[F(x)+F(x-)] F(x-) f(x)-6 F(x) F(x-) f(x)+3[F(x)+F(x-)] f(x) F(x-) \\
= & 6 F(x) f(x)-3 F(x) f(x)-3 F(x-) f(x)-3 F^{2}(x) f(x)+F^{3}(x)-F^{3}(x-) \\
= & 3 f^{2}(x)-3 F^{2}(x) f(x)+f(x)\left[3 F^{2}(x)-3 F(x) f(x)+f^{2}(x)\right] \\
= & f^{3}(x)+3 f^{2}(x)[1-F(x)] .
\end{aligned}
$$

This result is obtained if $r=1, s=2$, and $n=3$ in equation (2.4.3) in David [23].

Furthermore, if $x_{1} \leq x_{2} \leq \cdots \leq x_{p}$, it should be written $\iint \cdots \int$ instead of $\int$ in (3), where $\iint \cdots \int$ is to be carried out over the region: $v_{\varsigma_{2}^{(1)}}^{(1)} \leq v_{\varsigma_{4}^{(1)}}^{(2)} \leq \cdots \leq v_{\varsigma_{2 p}^{(1)}}^{(p)}, F_{\varsigma_{2}^{(1)}}\left(x_{1}-\right) \leq v_{\varsigma_{2}^{(1)}}^{(1)} \leq$ $F_{\varsigma_{2}^{(1)}}\left(x_{1}\right), F_{\varsigma_{4}^{(1)}}\left(x_{2}-\right) \leq v_{\varsigma_{4}^{(1)}}^{(2)} \leq F_{\varsigma_{4}^{(1)}}\left(x_{2}\right), \ldots, F_{\varsigma_{2 \mathrm{p}}^{(1)}}\left(x_{p}-\right) \leq v_{\varsigma_{2 \mathrm{p}}^{(1)}}^{(p)} \leq F_{\varsigma_{2 \mathrm{p}}^{(1)}}\left(x_{p}\right)$. 
We now express the following theorem to obtain the joint $d f$ of order statistics of innid discrete random variables.

\section{Theorem 3}

$$
\begin{aligned}
& F_{r_{1}, r_{2}, \ldots, r_{p}: n}\left(x_{1}, x_{2}, \ldots, x_{p}\right) \\
& =\sum_{z_{1}, z_{2}, \ldots, z_{p}} \sum_{m_{p}, k_{p}, \ldots, m_{1}, k_{1}} \sum_{n_{1}, n_{s_{2}}, \ldots, n_{s_{2} p}}\left(\prod_{w=1}^{p+1} \prod_{i_{2 w-1}=1}^{r_{w}-1-k_{w}-m_{w-1}-r_{w-1}}\left[F_{s_{2 w-1}\left(i_{2 w-1}\right)}\left(z_{w}-\right)-F_{s_{2 w-1}\left(i_{2 w-1}\right)}\left(z_{w-1}\right)\right]\right) \\
& \left.\quad \times \prod_{w=1}^{p} \prod_{i_{2 w}=1}^{k_{w}+1+m_{w}} f_{s_{2 w}} i_{\left.i_{2}\right)}\right)
\end{aligned}
$$

Proof We have

$$
F_{r_{1}, r_{2}, \ldots, r_{p}: n}\left(x_{1}, x_{2}, \ldots, x_{p}\right)=\sum_{z_{1}, z_{2}, \ldots, z_{p}} f_{r_{1}, r_{2}, \ldots, r_{p}: n}\left(z_{1}, z_{2}, \ldots, z_{p}\right)
$$

and using (1) in (8), (7) is obtained.

The identity (7) can also be written in the form of an integral as follows.

\section{Theorem 4}

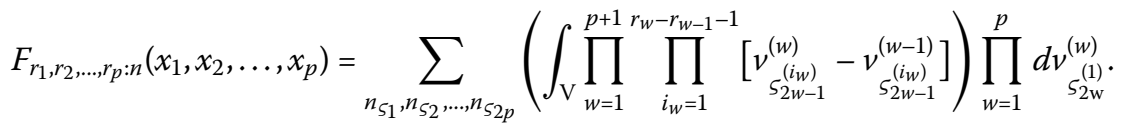

Proof Using (3) in (8), (9) is obtained.

\section{Results for distribution and probability functions}

In this section, the results related to $p f$ and $d f$ of $X_{r_{1}: n}, X_{r_{2}: n}, \ldots, X_{r_{p}: n}$ will be determined. We express the following result for $p f$ of the $r$ th order statistic of innid discrete random variables.

\section{Result 1}

$$
\begin{aligned}
f_{r_{1}: n}\left(x_{1}\right) & =\sum_{m_{1}=0}^{n-r_{1}} \sum_{k_{1}=0}^{r_{1}-1} \sum_{n_{s_{1}}, n_{s_{2}}}\left(\prod_{i_{1}=1}^{r_{1}-1-k_{1}} F_{\left.s_{1}^{\left(i_{1}\right.}\right)}\left(x_{1}-\right)\right)\left(\prod_{i_{2}=1}^{k_{1}+1+m_{1}} f_{s_{2}}^{\left(i_{2}\right)}\left(x_{1}\right)\right) \prod_{i_{3}=1}^{n-m_{1}-r_{1}}\left[1-F_{s_{3}}\left(i_{3}\right)\left(x_{1}\right)\right] \\
& =\sum_{n_{S_{1}}, n_{\zeta_{2}}} \int_{F_{s_{2}}^{(1)}\left(x_{1}-\right)}^{F} s_{2}^{(1)}\left(\prod_{i_{1}=1}^{\left(x_{1}\right)} v_{s_{1}^{\left(i_{1}\right)}}^{r_{1}-1}\right)\left(\prod_{i_{2}=1}^{n-r_{1}}\left[1-v_{s_{3}^{\left(i_{2}\right)}}^{(1)}\right)\right) d v_{s_{2}^{(1)}}^{(1)} .
\end{aligned}
$$

Proof In (1) and (3), if $p=1,(10)$ is obtained.

In Result 2 and Result 3, the pf's of minimum and maximum order statistics of innid discrete random variables are given respectively. 


\section{Result 2}

$$
\begin{aligned}
f_{1: n}\left(x_{1}\right) & =\sum_{m_{1}=0}^{n-1} \sum_{n_{s_{2}}}\left(\prod_{i_{2}=1}^{1+m_{1}} f_{s_{2}}\left(x_{1}\right)\right) \prod_{i_{3}=1}^{n-m_{1}-1}\left[1-F_{s_{3}\left(i_{3}\right)}\left(x_{1}\right)\right] \\
& =\sum_{n_{S 2}} \int_{F_{s_{2}^{(1)}}^{F} s_{2}^{(1)}\left(x_{1}\right)}^{\left(x_{1}\right)}\left(\prod_{i_{2}=1}^{n-1}\left[1-v_{s_{3}^{\left(i_{2}\right)}}^{(1)}\right]\right) d v_{s_{2}^{(1)}}^{(1)} .
\end{aligned}
$$

Proof In (10), if $r_{1}=1,(11)$ is obtained.

\section{Result 3}

$$
\begin{aligned}
f_{n: n}\left(x_{1}\right) & =\sum_{k_{1}=0}^{n-1} \sum_{n_{s_{1}}, n_{s_{2}}}\left(\prod_{i_{1}=1}^{n-1-k_{1}} F_{s_{1}^{\left(i_{1}\right)}}\left(x_{1}-\right)\right) \prod_{i_{2}=1}^{k_{1}+1} f_{s_{2}} f_{\left.i_{2}\right)}\left(x_{1}\right) \\
& =\sum_{n_{S_{1}}, n_{S_{2}}} \int_{F_{s_{2}}^{(1)} s_{2}^{(1)}\left(x_{1}\right)}^{\left(x_{1}\right)}\left(\prod_{i_{1}=1}^{n-1} v_{s_{1}^{\left(i_{1}\right)}}^{(1)}\right) d v_{s_{2}^{(1)}}^{(1)} .
\end{aligned}
$$

Proof In (10), if $r_{1}=n$, (12) is obtained.

In the following result, we determine the joint $p f$ of $X_{1: n}, X_{2: n}, \ldots, X_{p: n}$.

Result 4 If $x_{1} \leq x_{2} \leq \cdots \leq x_{p}$

$$
\begin{aligned}
& f_{1,2, \ldots, p: n}\left(x_{1}, x_{2}, \ldots, x_{p}\right)=\sum_{m_{p}=0}^{n-p} \sum_{n_{s_{2}}, n_{s_{4}}, \ldots, n_{s_{2 p}}}\left(\prod_{i_{2 p+1}=1}^{n-m_{p}-p}\left[1-F_{s_{2 p+1}}\left(i_{2 p+1}\right)\right]\right) \prod_{w=1}^{p} \prod_{i_{2 w}=1}^{1+m_{w}} f_{s_{2 w}}\left(i_{2 w}\right)\left(x_{w}\right) \\
& =\sum_{n_{\varsigma_{2}}, n_{\varsigma_{4}}, \ldots, n_{S_{2 p}}} \iint \cdots \int\left(\prod_{i_{p+1}=1}^{n-p}\left[1-v_{\varsigma_{2 p+1}^{(p)}}^{(p)}\right]\right) \prod_{w=1}^{p} d v_{\varsigma_{2 \mathrm{w}}}^{(w)} \text {, }
\end{aligned}
$$

where $\iint \cdots \int$ is to be carried out over the region: $v_{s_{2}^{(1)}}^{(1)} \leq v_{S_{4}^{(1)}}^{(2)} \leq \cdots \leq v_{S_{2 p}}^{(p)}, F_{s_{2}^{(1)}}\left(x_{1}-\right) \leq$ $v_{\varsigma_{2}^{(1)}}^{(1)} \leq F_{\varsigma_{2}^{(1)}}\left(x_{1}\right), F_{\varsigma_{4}^{(1)}}\left(x_{2}-\right) \leq v_{\varsigma_{4}^{(1)}}^{(2)} \leq F_{\varsigma_{4}^{(1)}}\left(x_{2}\right), \ldots, F_{\varsigma_{2 \mathrm{p}}^{(1)}}\left(x_{p}-\right) \leq v_{\varsigma_{2 \mathrm{p}}^{(1)}}^{(p)} \leq F_{\varsigma_{2 \mathrm{p}}^{(1)}}\left(x_{p}\right)$.

Proof In (1) and (3), if $r_{1}=1, r_{2}=2, \ldots, r_{p}=p$ and $\iint \cdots \int$ instead of $\int,(13)$ is obtained.

Specially, in (13), by taking $p=2, n=3$ and $v_{\varsigma_{5}^{(1)}}^{(2)}=\left[v_{\varsigma_{4}^{(1)}}^{(2)}-F_{\varsigma_{4}^{(1)}}\left(x_{2}-\right)\right] \frac{f_{\varsigma_{5}^{(1)}}^{\left(x_{2}\right)}}{f_{\varsigma_{4}^{(1)}}^{\left(x_{2}\right)}}+F_{\varsigma_{5}^{(1)}}\left(x_{2}-\right)$, the following identity is obtained

$$
\begin{aligned}
f_{1,2: 3}\left(x_{1}, x_{2}\right)= & \sum_{n_{s_{2}}, n_{s_{4}}}\left(1-F_{s_{5}^{(1)}}\left(x_{2}\right)\right) f_{s_{2}^{(1)}}\left(x_{1}\right) f_{s_{4}^{(1)}}\left(x_{2}\right)+\sum_{n_{s_{2}}} f_{s_{2}^{(1)}}\left(x_{1}\right) f_{s_{4}^{(1)}}\left(x_{2}\right) f_{s_{4}^{(2)}}\left(x_{2}\right) \\
= & \left(1-F_{1}\left(x_{2}\right)\right) f_{2}\left(x_{1}\right) f_{3}\left(x_{2}\right)+\left(1-F_{1}\left(x_{2}\right)\right) f_{3}\left(x_{1}\right) f_{2}\left(x_{2}\right)+\left(1-F_{2}\left(x_{2}\right)\right) f_{3}\left(x_{1}\right) f_{1}\left(x_{2}\right) \\
& +\left(1-F_{2}\left(x_{2}\right)\right) f_{1}\left(x_{1}\right) f_{3}\left(x_{2}\right)+\left(1-F_{3}\left(x_{2}\right)\right) f_{1}\left(x_{1}\right) f_{2}\left(x_{2}\right) \\
& +\left(1-F_{3}\left(x_{2}\right)\right) f_{2}\left(x_{1}\right) f_{1}\left(x_{2}\right)+f_{1}\left(x_{2}\right) f_{2}\left(x_{2}\right) f_{3}\left(x_{1}\right) \\
& +f_{2}\left(x_{2}\right) f_{1}\left(x_{2}\right) f_{3}\left(x_{1}\right)+f_{3}\left(x_{2}\right) f_{1}\left(x_{2}\right) f_{2}\left(x_{1}\right) .
\end{aligned}
$$


Moreover, the above identity in the iid case can be expressed as

$$
f_{1,2: 3}\left(x_{1}, x_{2}\right)=6 f\left(x_{1}\right) f\left(x_{2}\right)-6 f\left(x_{1}\right) f\left(x_{2}\right) F\left(x_{2}\right)+3 f\left(x_{1}\right) f^{2}\left(x_{2}\right) .
$$

We now establish three results for the $d f$ of single order statistic of innid discrete random variables.

\section{Result 5}

$$
\begin{aligned}
& F_{r_{1}: n}\left(x_{1}\right)=\sum_{z_{1}=0}^{x_{1}} \sum_{m_{1}=0}^{n-r_{1}} \sum_{k_{1}=0}^{r_{1}-1} \sum_{s_{1}, n_{s_{2}}}\left(\prod_{i_{1}=1}^{r_{1}-1-k_{1}} F_{s_{1}^{\left(i_{1}\right)}}\left(z_{1}-\right)\right)\left(\prod_{i_{2}=1}^{k_{1}+1+m_{1}} f_{s_{2}}\left(i_{2}\right)\left(z_{1}\right)\right) \prod_{i_{3}=1}^{n-m_{1}-r_{1}}\left[1-F_{s_{3}\left(i_{3}\right)}\left(z_{1}\right)\right] \\
& =\sum_{n_{S_{1}}, n_{S_{2}}} \int_{0}^{F_{s_{2}}^{(1)}}{ }^{\left(x_{1}\right)}\left(\prod_{i_{1}=1}^{r_{1}-1} v_{s_{1}^{\left(i_{1}\right)}}^{(1)}\right)\left(\prod_{i_{2}=1}^{n-r_{1}}\left[1-v_{s_{3}^{\left(i_{2}\right)}}^{(1)}\right]\right) d v_{s_{2}^{(1)}}^{(1)} \text {. }
\end{aligned}
$$

Proof $\operatorname{In}(7)$ and (9), if $p=1,(14)$ is obtained.

\section{Result 6}

$$
\begin{aligned}
F_{1: n}\left(x_{1}\right) & =\sum_{z_{1}=0}^{x_{1}} \sum_{m_{1}=0}^{n-1} \sum_{n_{s_{2}}}\left(\prod_{i_{2}=1}^{1+m_{1}} f_{s_{2}}\left(i_{2}\right)\left(z_{1}\right)\right) \prod_{i_{3}=1}^{n-m_{1}-1}\left[1-F_{s_{3}^{\left(i_{3}\right.}}\left(z_{1}\right)\right] \\
& =\sum_{n_{52}} \int_{0}^{F} s_{2}^{(1)}\left(\prod_{1}\right)\left(\prod_{i_{2}=1}^{n-1}\left[1-v_{\varsigma_{3}^{\left(i_{2}\right)}}^{(1)}\right]\right) d v_{\varsigma_{2}^{(1)}}^{(1)} .
\end{aligned}
$$

Proof In (14), if $r_{1}=1,(15)$ is obtained.

\section{Result 7}

$$
\begin{aligned}
F_{n: n}\left(x_{1}\right) & =\sum_{z_{1}=0}^{x_{1}} \sum_{k_{1}=0}^{n-1} \sum_{n_{s_{1}}, n_{s_{2}}}\left(\prod_{i_{1}=1}^{n-1-k_{1}} F_{s_{1}}\left(i_{1}\right)\right. \\
& =\sum_{n_{S 1}, n_{S 2}} \int_{0}^{F_{S_{2}}}{ }^{(1)} \prod_{i_{2}=1}^{k_{1}+1} f_{s_{2}}^{\left(i_{1}\right)}\left(\prod_{i_{1}}^{n-1} v_{i_{1}=1}^{(1)} v_{1}^{\left(i_{1}\right)}\right) d v_{s_{2}^{(1)}}^{(1)} .
\end{aligned}
$$

Proof In (14), if $r_{1}=n$, (16) is obtained.

\section{Competing interests}

The authors declare that they have no competing interests.

\section{Authors' contributions}

MG, YB and BY have contributed to all parts of the article. All authors read and approved the final manuscript.

\section{Author details}

${ }^{1}$ Department of Econometrics, Inonu University, Malatya, 44280, Turkey. ${ }^{2}$ Department of Mathematics, Bingol University,

Bingol, 12000, Turkey. 


\section{References}

1. Vaughan, RJ, Venables, WN: Permanent expressions for order statistics densities. J. R. Stat. Soc. B 34, 308-310 (1972)

2. Balakrishnan, N: Permanents order statistics, outliers and robustness. Rev. Mat. Complut. 20, 7-107 (2007)

3. Bapat, RB, Beg, MI: Order statistics for nonidentically distributed variables and permanents. Sankhya, Ser. A 51, 79-93 (1989)

4. Balasubramanian, K, Beg, MI, Bapat, RB: On families of distributions closed under extrema. Sankhya, Ser. A 53, 375-388 (1991)

5. Balasubramanian, K, Beg, MI, Bapat, RB: An identity for the joint distribution of order statistics and its applications. J. Stat. Plan. Inference 55, 13-21 (1996)

6. Cao, G, West, M: Computing distributions of order statistics. Commun. Stat., Theory Methods 26, 755-764 (1997)

7. Childs, A, Balakrishnan, N: Relations for order statistics from non-identical logistic random variables and assessment of the effect of multiple outliers on bias of linear estimators. J. Stat. Plan. Inference 136, 2227-2253 (2006)

8. Balasubramanian, K, Balakrishnan, N, Malik, HJ: Identities for order statistics from non-independent non-identical variables. Sankhya, Ser. B 56, 67-75 (1994)

9. Beg, MI: Recurrence relations and identities for product moments of order statistics corresponding to nonidentically distributed variables. Sankhya, Ser. A 53, 365-374 (1991)

10. Cramer, E, Herle, K, Balakrishnan, N: Permanent expansions and distributions of order statistics in the INID case. Commun. Stat., Theory Methods 38, 2078-2088 (2009)

11. Rath, D, Tripathy, BC: Matrix maps on sequence spaces associated with sets of integers. Indian J. Pure Appl. Math. 27(2), 197-206 (1996)

12. Tripathy, BC: Matrix transformations between some classes of sequences. J. Math. Anal. Appl. 206, $448-450$ (2001)

13. Tripathy, BC: On generalized difference paranormed statistically convergent sequences. Indian J. Pure Appl. Math. 35(5), 655-663 (2004)

14. Tripathy, BC, Baruah, A: Lacunary statistical convergent and lacunary strongly convergent generalized difference sequences of fuzzy real numbers. Kyungpook Math. J. 50(4), 565-574 (2010)

15. Tripathy, BC, Dutta, H: On some lacunary difference sequence spaces defined by a sequence of Orlicz functions and $q$-lacunary $\Delta_{m}^{n}$-statistical convergence. An. Stiint. Univ. Ovidius, Ser. Mat. 20(1), 417-430 (2012)

16. Tripathy, BC, Sarma, B: Statistically convergent difference double sequence spaces. Acta Math. Sin. 24(5), 737-742 (2008)

17. Tripathy, BC, Sen, M: On generalized statistically convergent sequences. Indian J. Pure Appl. Math. 32(11), 1689-1964 (2001)

18. Corley, HW: Multivariate order statistics. Commun. Stat., Theory Methods 13, 1299-1304 (1984)

19. Guilbaud, O: Functions of non-i.i.d. random vectors expressed as functions of i.i.d. random vectors. Scand. J. Stat. 9 229-233 (1982)

20. Goldie, CM, Maller, RA: Generalized densities of order statistics. Stat. Neerl. 53, 222-246 (1999)

21. Arnold, BC, Balakrishnan, N, Nagaraja, HN: A First Course in Order Statistics. Wiley, New York (1992)

22. Balasubramanian, K, Beg, Ml: On special linear identities for order statistics. Statistics 37, 335-339 (2003)

23. David, HA: Order Statistics. Wiley, New York (1970)

24. Reiss, R-D: Approximate Distributions of Order Statistics. Springer, New York (1989)

25. Gan, G, Bain, LJ: Distribution of order statistics for discrete parents with applications to censored sampling. J. Stat. Plan. Inference 44, 37-46 (1995)

26. Khatri, CG: Distributions of order statistics for discrete case. Ann. Inst. Stat. Math. 14, 167-171 (1962)

27. Balakrishnan, N: Order statistics from discrete distributions. Commun. Stat., Theory Methods 15, $657-675$ (1986)

28. Nagaraja, HN: Structure of discrete order statistics. J. Stat. Plan. Inference 13, 165-177 (1986)

29. Nagaraja, HN: Order statistics from discrete distributions. Statistics 23, 189-216 (1992)

\section{Submit your manuscript to a SpringerOpen ${ }^{0}$ journal and benefit from:}

- Convenient online submission

- Rigorous peer review

- Immediate publication on acceptance

- Open access: articles freely available online

- High visibility within the field

- Retaining the copyright to your article

Submit your next manuscript at $>$ springeropen.com 